\title{
A Role for Endothelin-2 and Its Receptors in Breast Tumor Cell Invasion
}

\author{
Matthew J. Grimshaw, ${ }^{1,4}$ Thorsten Hagemann, ${ }^{2}$ Ayse Ayhan, ${ }^{3}$ Cheryl E. Gillett, ${ }^{5}$ Claudia Binder, ${ }^{2}$ and \\ Frances R. Balkwill ${ }^{1}$

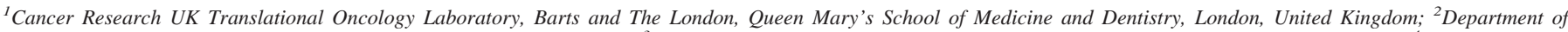

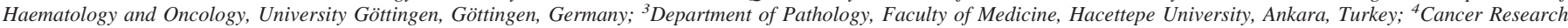 \\ UK Breast Cancer Biology Group, Guy's Hospital, and ${ }^{5}$ Hedley Atkins/Cancer Research UK Breast Pathology Laboratory, Guy's Hospital, London, United Kingdom
}

\section{ABSTRACT}

We have studied the role of endothelins (ET-1, ET-2 and ET-3) and ET receptors (ET-RA and ET-RB) in the invasive capacity of breast tumor cells, which express ET-1 and ET-2 as well as ET-RA and ET-RB. Of five human breast tumor cell lines tested, all expressed mRNAs for ET-1, ET-2, and ET-RB. ET-RA mRNA was expressed by four of five tumor cell lines. Breast tumor cells migrated toward ET-1 and ET-2 but not toward ET-3. Chemotaxis involved signaling via both receptors, and a pertussis toxin-sensitive $\mathbf{p}$ 2/p44 mitogen-activated protein kinase (MAPK)mediated pathway that could be inhibited by MAPK kinase (MEK)1/2 antagonists. Chemotaxis toward ETs did not involve p38 or stressactivated protein kinase (SAPK)/Jun N-terminal kinase (JNK) and was not inhibited by hypoxia. Incubation of tumor cells with ET-2 also increased chemotaxis toward the chemokines CXCL12 and CCL21. As well as inducing chemotaxis of tumor cells, ET-1 and ET-2 increased tumor cell invasion through Matrigel. Furthermore, stimulation of macrophage/tumor cell cocultures with ETs led to increased matrix metalloproteinase (MMP)-2 and -9 production by macrophages and a marked increase in invasion of tumor cells. Antagonism of either ET-RA or ET-RB decreased the invasion seen in ET-stimulated cocultures, as did a broad-spectrum MMP inhibitor. Immunohistochemical staining of human breast tumor sections showed increased ET and ET receptor protein expression by tumor cells in invasive ductal carcinoma compared with normal breast tissue or ductal carcinoma in situ. Furthermore, tumor cell ET and receptor expression was stronger at the invasive margin of invasive ductal carcinomas, in the lymphovascular space, and in lymph node metastases. ET expression often colocalized with ET-RB expression in all neoplastic tissue indicating a possible autocrine action of ETs. We suggest that expression of ETs and their receptors by human breast tumors, particularly in conjunction with a high macrophage infiltrate, may have a role in the progression of breast cancer and the invasion of tumor cells.

\section{INTRODUCTION}

Endothelins (ETs) are a group (ET-1, ET-2 and ET-3) of small (21-amino acid) vasoactive peptides with diverse paracrine/autocrine actions (1). ET-1 and ET-2 have similar structures, differing by only two amino acids, whereas ET-3 differs in structure at six amino acids (2). ETs bind at least two receptor subtypes, ET-A receptors (ET-RA) and ET-B receptors (ET-RB; Ref. 3), which are seven-transmembrane domain $\mathrm{G}$ protein-coupled receptors (4).

ETs are expressed in a broad range of tumors, and there is a growing interest in ETs and cancer; most reports focus on the role of ET-1/ET-RA in tumor growth, progression, and angiogenesis (5). However, we recently proposed a role for ET-2/ET-RB in invasive ductal carcinoma (IDC) of the breast: binding of ET-2 to ET-RB protects tumor cells from hypoxia-associated apoptosis in an autocrine fashion (6). Both ET-2 and ET-RB are rarely detected in the nonneoplastic human breast, but breast carcinomas contain regions of hy-

Received 4/17/03; revised 1/19/04; accepted 1/30/04

The costs of publication of this article were defrayed in part by the payment of page charges. This article must therefore be hereby marked advertisement in accordance with 18 U.S.C. Section 1734 solely to indicate this fact.

Requests for reprints: Matthew Grimshaw, Cancer Research United Kingdom, Breas Cancer Biology Group, 3rd Floor, Thomas Guy House, Guy's Hospital, London SE1 9RT, United Kingdom. Phone: 00-44-20-7955-2335; Fax: 00-44-20-7955-2027; E-mail: matthew.grimshaw@ cancer.org.uk. poxia that increase production of ET-2 as well as ET-RA and ET-RB by tumor cells (6). Intratumoral injection of BQ-788, an ET-RB selective antagonist (7), causes increased necrosis and decreased tumor volume in a murine model of breast carcinoma (6); BQ-123, a selective ET-RA antagonist (8), also leads to decreased breast tumor volume but without a concomitant increase in necrosis.

High levels of macrophage infiltrate in breast tumors correlate with a poor prognosis (9) and hypoxic tumors have a higher invasive capacity and a poorer prognosis than well-oxygenated tumors (10). Hypoxia-associated ETs expression by tumor cells may also modulate tumor-associated macrophage distribution and activation in breast tumors; ET-1 and ET-2, but not ET-3, are chemoattractant for macrophages and lead to an activated phenotype in vitro (11). In vivo, ET expression in IDC not only correlates with hypoxia (6) but also with the presence of activated "foamy" tumor-associated macrophages (11).

ETs may also play a role in chemotaxis of several additional cell types: ET-1 and ET-3 are chemoattractants for pulmonary artery fibroblasts (12), and ET-2 is a chemoattractant for neutrophils (13).

As well as being chemoattractant for several cell types, ET-1 may also modulate the invasive capacity of some tumor cells including ovarian carcinoma cells (14), Ewing's sarcoma, and neuroblastoma cells (15). In ovarian carcinoma cells, ET-1 interacts with ET-RA to increase the expression and activation of matrix metalloproteinases (MMPs) whereas decreasing the secretion of tissue inhibitor(s) of MMPs (TIMP; Ref. 14).

In view of the possible role of ETs in invasion of tumor cells, we investigated whether ETs are chemoattractant for breast carcinoma cells and, in conjunction with macrophages, whether they may modulate the invasive capacity of breast tumor cells. By examining human neoplastic breast tissues, we also analyzed whether ETs are associated with progression and invasion of tumor cells in ductal carcinoma in situ (DCIS), IDC, and lymph node metastases. Because of its regulation by hypoxia and increased expression in IDC compared with normal breast tissue (6), we focused on the potential role of ET-2/ ET-RB.

\section{MATERIALS AND METHODS}

Peptides and Antagonists. ET peptides and receptor antagonists BQ-123 (ET-RA antagonist; Ref. 16) and BQ-788 (ET-RB antagonist; Ref. 7) were obtained from American Peptide Co., Sunnyvale, CA. MAPK kinase (MEK)1/2 inhibitors PD98059 and U0126 were purchased from Cell Signaling Technology Inc., Beverly, MA. Pertussis toxin (PTX; G-protein inhibitor) and FN439 (nonspecific MMP inhibitor) were supplied by Calbiochem-Novabiochem Corporation, San Diego, CA.

Cell Culture. HTH-K cells were cultured as described previously (17). Human cell lines (MCF-7, BT20, BT474, SKBR3, and MDAMB468) from the American Type Culture Collection (Manassas, VA) were cultured similarly. peripheral blood mononuclear cells were isolated from human blood and matured into macrophages under endotoxin-free conditions as described previously (11).

Reverse Transcription-PCR. RNA was isolated from cell lines, and cDNA was amplified as described previously (6).

Microchemotaxis Assay. Subconfluent tumor cells were trypsinized and resuspended in DMEM supplemented with $10 \%$ FCS $\left(2 \times 10^{5}\right.$ cells $\left./ \mathrm{ml}\right)$ and were 
then subjected to chemotaxis assays and counted as described previously (11). A $12-\mu \mathrm{m}$ membrane, that had been treated with $6.5 \mu \mathrm{g} / \mathrm{ml}$ fibronectin solution at $37^{\circ} \mathrm{C}$ for $2 \mathrm{~h}$ and then dried was used. Migration was stopped after $5 \mathrm{~h}$ for each experiment.

Western Blotting. Protein extracts were prepared from cells that had been serum-starved for $24 \mathrm{~h}$ before stimulation with ETs. Western blotting for ET receptors was carried out as described previously (6) and for signaling pathways as described previously (11).

Matrigel Invasion Assay. In vitro-invasiveness was measured in a modified Boyden chamber as described in Hagemann et al. ${ }^{6} \mathrm{MCF}-7$ cells were seeded in gelatinase-free medium on polycarbonate membranes coated with Matrigel (ECM gel; Sigma-Aldrich Co. Ltd., Poole, United Kingdom). Macrophages were added at 2:1 (macrophages:tumor cells) in inserts that allow exchange of liquids but no direct cell-cell contact or invasion of macrophages. ETs $(100 \mathrm{ng} / \mathrm{ml})$ were added, and renewed every $24 \mathrm{~h}$. After $96 \mathrm{~h}$, the contents of the lower well were removed, and cells were collected by centrifugation. The supernatant was used for zymography. Cells were spun down onto coverslips and stained with 4',6'-diamino-2'-phenylindol (DAPI, $200 \mathrm{ng} / \mathrm{ml}$ ). Intact nuclei were counted in six high power fields using UV-microscopy (Axioskop; Zeiss, Jena, Germany).

Zymography. Protein content in the supernatant was determined using the bicinchoninic acid assay (Pierce, Rockford, IL). Twenty $\mu \mathrm{g}$ were separated on $10 \%$ SDS-polyacrylamide gels containing $1 \mathrm{mg} / \mathrm{ml}$ gelatin. After electrophoresis, gels were washed for $1 \mathrm{~h}$ in renaturation buffer $(2.5 \%$ Triton $\mathrm{X}-100)$ and then were incubated for $36 \mathrm{~h}$ at $37^{\circ} \mathrm{C}$ in $50 \mathrm{~mm}$ Tris, $200 \mathrm{~mm} \mathrm{NaCl}$, and $5 \mathrm{~mm}$ $\mathrm{CaCl}_{2}$ (pH 7.5). Gels were stained with $0.05 \%$ Coomassie Brilliant Blue, and MMPs were identified by size and comparison with the recombinant proteins (Calbiochem-Novabiochem Corporation).

Immunohistochemistry of Human Breast Tumor Samples. Intraductal and invasive human breast cancer archival samples and corresponding metastatic lymph node samples were supplied by the Hedley Atkins Breast Pathology Laboratory, Guy's Hospital, London, United Kingdom. All of the specimens were formalin-fixed and paraffin-embedded. Protein expression of ETs and ET receptors were immunohistochemically examined as previously (11). Sections from representative paraffin blocks of tumors were deparaffinized, rehydrated, and blocked for endogenous peroxidase activity using $3 \% \mathrm{H}_{2} \mathrm{O}_{2}$. Mature ETs were detected using a rabbit antimouse ET polyclonal antibody (Oncogene Research Products, Cambridge, MA) that recognizes all ET isoforms (18). Sections were also immunostained with antibodies raised against the $\mathrm{COOH}$ terminus of the ET receptors (19). Sections were stained as described previously (11) using a sheep anti-ET-RA polyclonal antibody (Alexis Biochemicals, San Diego, CA) or a sheep anti-ET-RB polyclonal antibody (Calbiochem-Novabiochem Corporation). The specificity of all antibodies used was proven using blocking peptides by the supplying companies (data not shown). Appropriate biotinylated secondary antibodies were used. The sections were incubated with streptavidin-peroxidase and were developed using diaminobenzidene substrate followed by counterstaining with Harris's hematoxylin. The sections were independently examined by a consultant histopathologist (A. A.). Immunoreactivity was semiquantitatively evaluated by comparing the level of expression in malignant cells with that of normal breast tissue present in the same section. Tumors were considered to be "weakly positive" if up to $25 \%$ of cells expressed the protein and "strongly positive" if more than $25 \%$ of cells did. This cutoff point was chosen because it seemed to divide the sections into two distinct groups. No staining was observed with negative control samples (absence of primary antibody, or incubation with an irrelevant antibody or $\mathrm{IgG})$.

Statistical Analysis. Results were tested for statistical significance using one-way ANOVA or Tukey-Kramer multiple comparisons test with InStat Version 3.0 software. Western blots were quantified by densitometry using NIH Image Version 1.63. ${ }^{7}$

${ }^{6}$ T. Hagemann, S. C. Robinson, M. Schulz, L. Trumper, F. R. Balkwill, and C. Binder. Enhanced invasiveness of breast cancer cell lines upon cocultivation with macrophages is due to TNF- $\alpha$-dependent up-regulation of matrix metalloproteases., submitted for publication.

${ }^{7}$ URL: http://rsb.info.nih.gov/nih-image/

\section{RESULTS}

ET and ET Receptor mRNA Expression by Human Breast Tumor Cell Lines. The murine breast carcinoma cell line HTH-K expresses ET-2, ET-RA, and ET-RB, and this expression is increased by exposure to hypoxia (6). We examined ET and ET receptor mRNA expression in five human breast tumor cell lines: SKBR3, MCF-7, BT20, BT474, and MDAMB468. Similar to the expression profile seen in IDC of the breast (6), all of the tumor cell lines expressed ET-1 and ET-2, but none expressed ET-3; all of the cell lines expressed ET-RB, whereas all but one, BT20, expressed ET-RA (Fig. 1A).

Breast Tumor Cells Chemotax toward ET-2. We used breast carcinoma cell lines to investigate chemotaxis of breast tumor cells toward ETs in vitro. MCF-7 cells migrated strongly toward $100 \mathrm{ng} / \mathrm{ml}$ ET-2 and less robustly toward ET-1 (Fig. 1B). Tumor cells did not

A

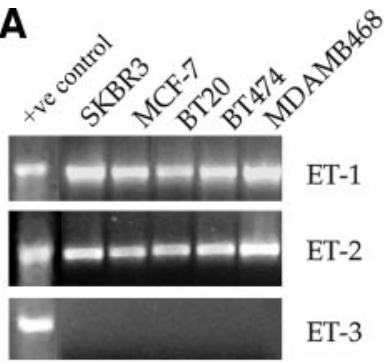

B

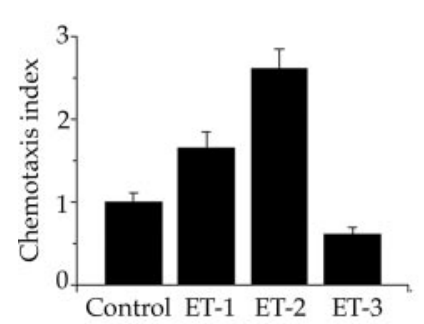

D

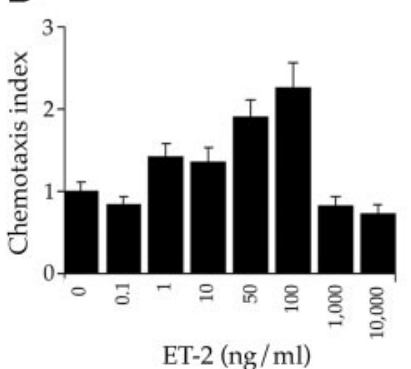

Fig. 1. A, reverse transcription-PCR (RT-PCR) screen for endothelin (ET-1, ET-2, $E T-3)$ and endothelin receptor (ET-RA and $E T-R B)$ mRNA expression by human breast cancer cell lines. Positive controls are RT-PCR products from RNA derived from human tissues known to express ETs $(1,6)$. B, a 5-h microchemotaxis assay of MCF-7 cells toward $100 \mathrm{ng} / \mathrm{ml}$ endothelin. Chemotaxis toward ET-1 and ET-2 was significantly increased compared with control $(P<0.05$ and $P<0.01$, respectively). $C$, microchemotaxis assay of breast tumor cells lines toward ET-2 $(100 \mathrm{ng} / \mathrm{ml})$. ET-2 significantly increased migration of HTH-K cells $(P<0.01)$, MDAMB468 $(P<0.01)$, and SKBR3 $(P<0.01)$. BT20 migrated weakly but significantly toward ET-2 $(P<0.05)$. D, doseresponse curve of MCF-7 cell chemotaxis toward ET-2. The maximum number of migrated cells per high power field (HPF) and greatest significance $(P<0.01)$ was at $100 \mathrm{ng} / \mathrm{ml} \mathrm{ET}-2$. E, gradient-independent migration and ET-2. Chemotaxis of MCF-7 cells was measured when $100 \mathrm{ng} / \mathrm{ml}$ ET-2 was present in the lower chamber (ET-2) or both upper and lower chambers $(E T-2 / E T-2)$. Chemotaxis toward ET-2 was abolished by the addition of ET-2 to the upper chamber $(P<0.001)$. Microchemotaxis assay results are expressed as the mean $( \pm \mathrm{SE})$ of number of cells per HPF/control cells per HPF. Shown is one experiment (of at least three) and $n=8$ microchemotaxis assay wells per parameter. 

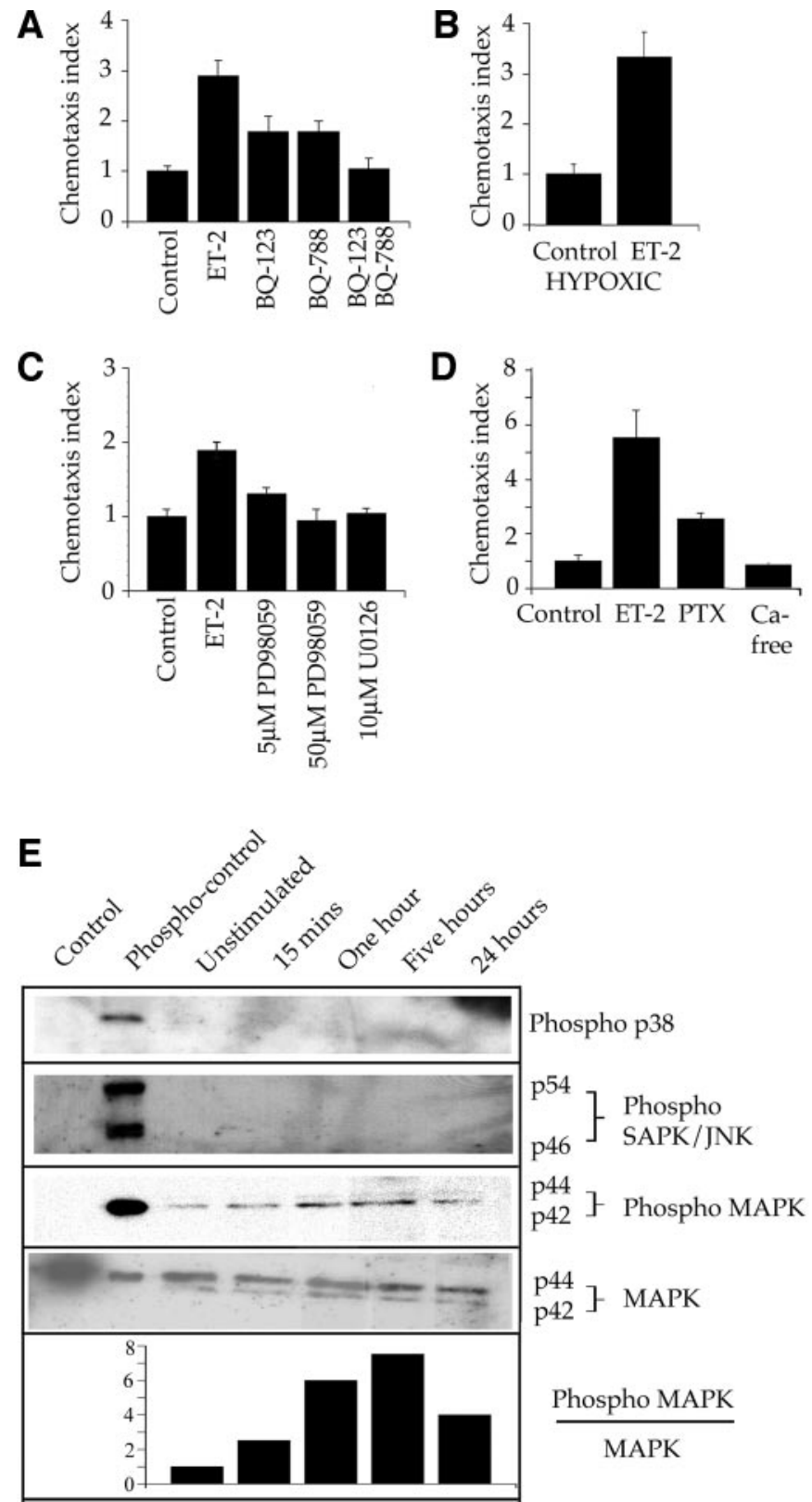

Fig. 2. A, effect of endothelin (ET) receptor inhibition on tumor cell chemotaxis toward ET-2. MCF-7 cells were incubated with $100 \mathrm{ng} / \mathrm{ml}$ BQ-123 or BQ-788 (singly and in combination) for $30 \mathrm{~min}$ before and during the microchemotaxis assays toward $100 \mathrm{ng} / \mathrm{ml}$ ET-2. Both BQ-123 and BQ-788 could partially but significantly inhibit migration toward ET-2 $(P<0.001)$. BQ-123 and BQ-788 together abolished chemotaxis $(P<0.001)$. $B$, chemotaxis of MCF-7 cells toward ET-2 under hypoxia. The microchemotaxis chamber was incubated at $0.1 \%$ oxygen for the duration of the assay. During hypoxia, MCF-7 cells were still able to migrate toward ET-2 $(P<0.05)$. $C$, inhibition of chemotaxis toward ET-2 by mitogen-activated protein kinase $(M A P K)$ kinase (MEK) $1 / 2$ inhibitors. MCF-7 cells were incubated in $5 \mu \mathrm{M}$ PD98059, $50 \mu \mathrm{M}$ PD98059, or $10 \mu \mathrm{M}$ U0126 for $30 \mathrm{~min}$ before and during the microchemotaxis assay to $100 \mathrm{ng} / \mathrm{ml}$ ET-2. Migration was partially inhibited by $5 \mu \mathrm{M}$ PD98059 $(P<0.001)$ and completely abolished by $50 \mu \mathrm{M}$ PD98059 $(P<0.05)$ or $10 \mu \mathrm{M}$ U0126 $(P<0.05)$. $D$, inhibition of chemotaxis toward ET-2 by inhibition of $\mathrm{G}$ proteins or by incubation in calcium-free medium ( $\mathrm{Ca}$-free). MCF-7 cells were incubated in $100 \mathrm{ng} / \mathrm{ml}$ pertussis toxin $(P T X)$ or in Ca-free medium for $30 \mathrm{~min}$ before and during the microchemotaxis assay to $100 \mathrm{ng} / \mathrm{ml}$ ET-2. Migration was significantly inhibited by PTX $(P<0.001)$ or by incubation in Ca-free medium $(P<0.05)$. $E$, Western analysis of protein phosphorylation in MCF-7 cells on stimulation with ET-2. MCF-7 cells were serum-starved for $24 \mathrm{~h}$ before being stimulated with ET-2 for $0-24 \mathrm{~h}$. ET-2 led to a rapid (15 min) phosphorylation of p42/p44 MAPK (Phospho-MAPK). p38, p46, and p54 were not phosphorylated in response to ET-2. Shown is one experiment of three. Phosphorylated and nonphosphorylated controls were either treated cell extracts [p38 (Phospho 38), stress-activated protein kinase/Jun N-terminal kinase (Phospho Sapk/JNK)] or control peptides (p42/44 MAPK) supplied by the antibody manufacturer. The bottom panel shows a graph of the phospho-MAPK:total MAPK ratio (normalized to the unstimulated control), as assessed by densitometry, from a single representative experiment (of three). migrate toward ET-3. Murine HTH-K cells had a similar chemotactic profile, and the other human breast tumor cell lines tested (MDAMB468, SKBR3, and BT20) also chemotaxed toward ET-2 (Fig. 1C). BT20, which does not express ET-RA (Fig. 1A), had a decreased chemotactic ability toward ET-2, compared with other cell lines (Fig. 1C).

In a dose-response assay, MCF-7 cells migrated toward ET-2 demonstrating a bell-shaped response curve with maximum migration at $50-100 \mathrm{ng} / \mathrm{ml} \mathrm{ET-2} \mathrm{(Fig.} \mathrm{1D).} \mathrm{To} \mathrm{ensure} \mathrm{that} \mathrm{ET-2} \mathrm{did} \mathrm{not} \mathrm{stim-}$ ulate random migration, the ET-2 gradient was abolished by adding the peptide to both lower and upper wells of the chamber; this revealed that the effects of ET-2 are chemotactic rather than chemokinetic (Fig. 1E).

Chemotaxis to ET-2 Involves Both ET-RA and ET-RB. To determine which receptor(s) is responsible for tumor cell chemotaxis, we analyzed chemotaxis toward ET-2 in the presence of ET-RA antagonist BQ-123 (16) and/or ET-RB antagonist BQ-788 (7). At a concentration of $100 \mathrm{ng} / \mathrm{ml}$, these modified-peptide antagonists are highly specific for their respective receptor $(7,16)$ and can distinguish between ET-RA- and ET-RB-induced chemotaxis (11). Incubation of MCF-7 cells with either BQ-123 or BQ-788 before and during the microchemotaxis assay partially decreased, but did not eliminate, migration toward ET-2 (Fig. 2A). However, combining both antagonists abolished migration toward ET-2 (Fig. 2A). Neither of the ET receptor antagonists affected viability (assessed by trypan blue exclusion assay; data not shown).

ET-2 Signals via the p42/p44 MAPK Pathway in Breast Carcinoma Cells. We next analyzed the signaling pathways involved in ET-induced chemotaxis. In macrophages, chemotaxis toward ET-2 is via an ET-RB-mediated MAPK pathway that can be inhibited by hypoxia (11).

Microchemotaxis assays of MCF-7 cells toward ET-2 were carried out in a hypoxic incubator $\left(0.1 \%\right.$ oxygen, $10 \% \mathrm{CO}_{2}$, balance nitrogen). Unlike macrophages (11), breast tumor cells were not inhibited by hypoxia from migrating toward ET-2 (Fig. 2B).

PD98059, which inhibits MEK1 at low concentrations $(5 \mu \mathrm{M})$ and MEK1/2 at higher concentrations (50 $\mu \mathrm{M}$; Ref. 20), partially inhibited MCF-7 cell chemotaxis toward ET-2 at $5 \mu \mathrm{M}$ and completely blocked chemotaxis at $50 \mu \mathrm{M}$ (Fig. 2C). The MEK1/2 inhibitor U0126 (10 $\mu \mathrm{M})$ also inhibited migration of MCF-7 cells toward ET-2 (Fig. 2C).

ET-RA may increase intracellular calcium by at least two mechanisms: a PTX-insensitive calcium mobilization from intracellular stores and a PTX-sensitive influx of extracellular calcium (21). PTX

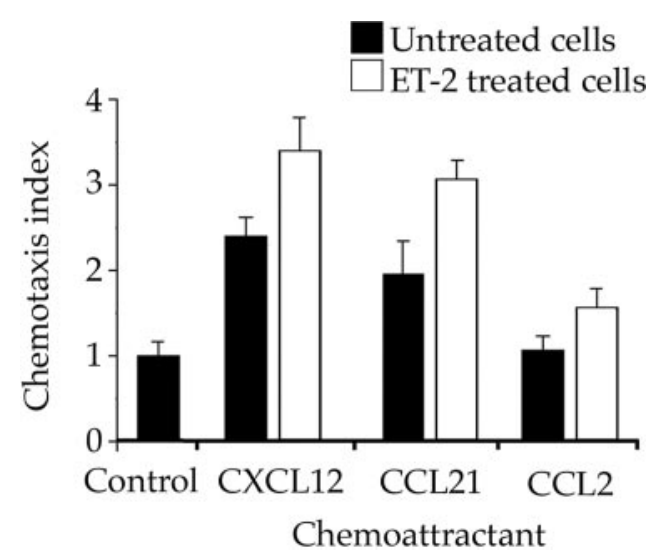

Fig. 3. Chemotaxis of MCF-7 cells toward $100 \mathrm{ng} / \mathrm{ml}$ CXCL12, CCL21, or CCL2 in the presence of $100 \mathrm{ng} / \mathrm{ml}$ endothelin (ET)-2. Chemotaxis of MCF-7 cells toward CXCL12 and CCL21 was significantly increased compared with control $(P<0.01)$, and this was further increased by the presence of ET-2 $(P<0.001)$ MCF-7 cells did not significantly migrate toward CCL2 with or without ET-2. 
A

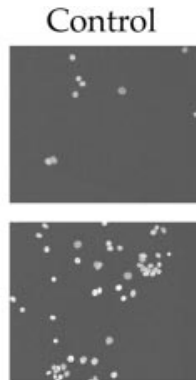

ET-2

B

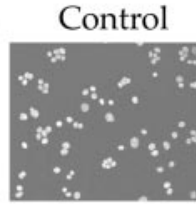

Fig. 4. A, invasion of MCF-7 cells toward endothelins (ETs). Chemotactic invasion of MCF-7 cells was tested in a 96-h invasion assay toward $100 \mathrm{ng} / \mathrm{ml}$ ET in the lower chamber. ETand ET-2 modestly but significantly $(P<0.05)$ increased invasion. ET-3 had no significant effect. $B$, gradient-independent invasion induced by ETs. MCF-7 cells were used in an invasion assay in which $100 \mathrm{ng} / \mathrm{ml}$ ET was added to the upper chamber. ET-1 and ET-2 significantly $(P<0.05)$ increased invasion. $C$, gradient-independent invasion of tumor cells induced by ETs in MCF-7/macrophage cocultures. MCF-7 cells and macrophages were used in an invasion assay with $100 \mathrm{ng} / \mathrm{ml} \mathrm{ET}$ in the upper chamber. Macrophages $(m \phi)$ increased invasion of MCF-7 cells $(P<0.01)$, and invasion was further increased by both ET-1 and ET-2 $(P<0.01)$. ET-3 had no effect on invasion of cocultured tumor cells. $D$, inhibition of ET-induced invasion in MCF-7/ macrophage coculture by ET receptor antagonists. One hundred $\mathrm{ng} / \mathrm{ml} \mathrm{BQ}-123$ and/or BQ-788 were added to coculture invasion assays stimulated with ET-2. Both BQ-123 and BQ-788 significantly reduced ET-2-stimulated invasion of MCF-7 cells in coculture $(P<0.05)$. The addition of both BQ-123 and BQ-788 did not decrease invasion more than either antagonist alone. Invasion assay results are the mean $( \pm \mathrm{SE})$ of the number of cells per-high power field/control cells per high-power field. Shown is one experiment (of three); $n \geq 12$.

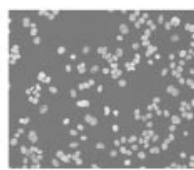

ET-2

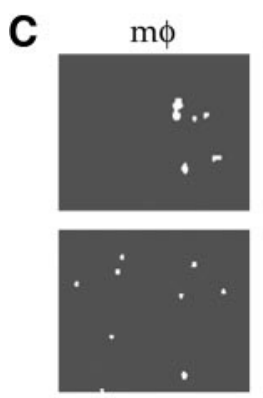

$\mathrm{m} \phi+\mathrm{ET}-2$

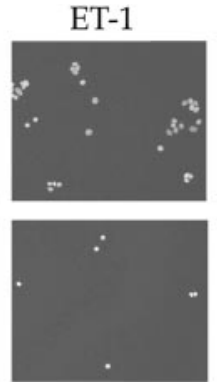

ET-3

\section{ET-1}

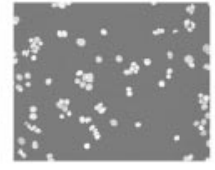

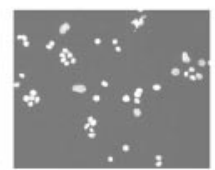

ET-3 $\mathrm{m} \phi+\mathrm{ET}-1$
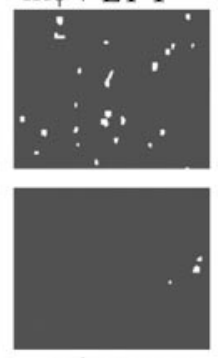

$\mathrm{m} \phi+\mathrm{ET}-3$

$\mathrm{m} \phi+\mathrm{ET}-2$
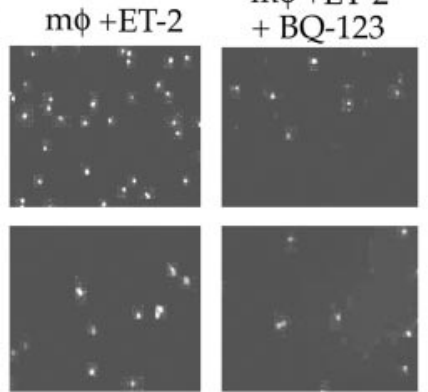

$\mathrm{m} \phi+\mathrm{ET}-2$

$+\mathrm{BQ}-788$

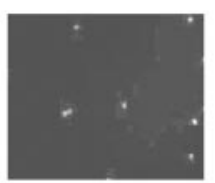

$\mathrm{m} \phi+\mathrm{ET}-2$

$+\mathrm{BQ}-123$

+ BQ-788
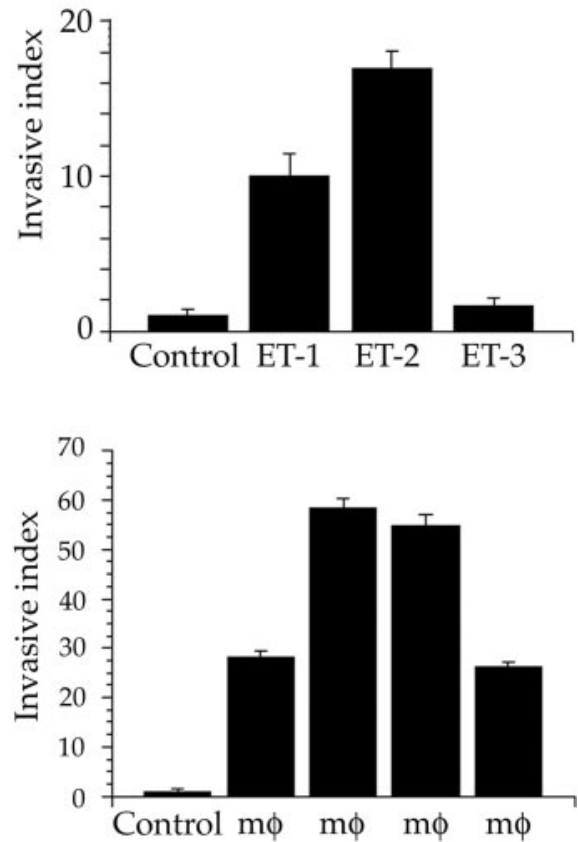

ET-1 ET-2 ET-3

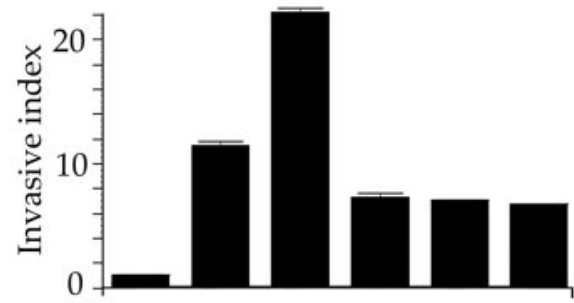

$\mathrm{m} \phi \quad-\quad+\quad+\quad+\quad+$

ET-2 - $\quad-\quad+\quad+\quad+\quad+$

$\mathrm{BQ}-123 \quad-\quad-\quad-\quad+\quad-\quad+$ also inhibits ET-RB-mediated chemotaxis of macrophages toward ET-2 (11). Incubation of MCF-7 cells with PTX (100 ng/ml) led to decreased migration toward ET-2 (Fig. 2D). Migration of MCF-7 cells toward ET-2 was also inhibited by incubating the cells in calcium-free medium before and during the chemotaxis assay (Fig. 2D).

Cell viability was not affected by any of the inhibitors used (data not shown).

To demonstrate that activation of the p42/p44 MAPK pathway was responsible for chemotaxis toward ETs, we analyzed the phosphorylation status of key molecules in several distinct signaling pathways.
Incubation of serum-starved MCF-7 cells with $100 \mathrm{ng} / \mathrm{ml}$ ET-2 for $0-24 \mathrm{~h}$ led to a transient activation of p42 and p44 MAPK with maximal phosphorylation between 1 and $5 \mathrm{~h}$ (Fig. 2E). There was no activation of the stress-activated p38 or stress-activated protein kinase (SAPK)/Jun N-terminal kinase (JNK) pathways (Fig. 2E).

ET-2 Increases Breast Carcinoma Cell Chemotaxis to Chemokines. Because chemokines may play a role in breast tumor metastasis (22), we analyzed the ability of tumor cells exposed to ET-2 to chemotax toward CXCL12 and CCL21. Primary breast carcinoma cells and cell lines, including MCF-7, express the chemokine recep- 
tors CXCR4 and CCR7, which mediate migration toward CXCL12 and CCL21, respectively (22). These chemokines are expressed at sites of metastasis such as the lymph nodes (22). Cells that had been incubated with ET-2 before the chemotaxis assay had a higher migratory capacity toward CXCL12 and CCL21 than did untreated cells (Fig. 3). MCF-7 cells do not express CCR2 (22), and ET-2 did not significantly increase migration toward CCL2.

We analyzed whether the increased migration toward the chemokines CXCL12 and CCL21 was due to up-regulation of the chemokine receptors CXCR4 and CCR7 respectively. Fluorescence-activated cell sorting analysis showed that there was no up-regulation of these receptors at the cell surface (data not shown).

ET-1 and ET-2 Increase MCF-7 Cell Invasion through Matrigel in Vitro. We next investigated whether ETs increase the invasive capacity of breast tumor cells in an in vitro invasion assay. To test whether ETs may induce invasion in a chemotactic manner, ET peptides were added to the lower wells of the invasion chamber during a $96 \mathrm{~h}$ assay. ET-1 and ET-2 caused an increase in invasion (Fig. 4A) whereas ET-3 had no significant effect.

Next, we analyzed whether incubating cells with ETs increases gradient-independent invasion. ET peptide $(100 \mathrm{ng} / \mathrm{ml})$ was added to the cells in the upper well of the invasion assay; ET-1 and ET-2 greatly increased the number of tumor cells that had invaded into the lower chamber (Fig. 4B), whereas ET-3 had no effect.

ET-1 and ET-2 Increase Invasion of Tumor Cells Cocultured with Macrophages. Coculture of breast tumor cells with macrophages leads to increased invasive capacity of tumor cells compared with tumor cells cultured in isolation (Hagemann et al.). ${ }^{6}$ Furthermore, incubation of macrophages with ET-2 leads to increased activation of these cells (11). Therefore, we evaluated the effect of ETs on cocultured macrophages/tumor cells. The presence of macrophages led to increased MCF-7 tumor cell invasion through Matrigel compared with tumor cells cultured in isolation (Fig. 4C). Addition of ET-1 or ET-2 to the cocultured cells led to a further increase in invasion by MCF-7 cells, but ET-3 had no effect (Fig. 4C).

To test which ET receptor(s) was involved in the increased invasion of cocultured MCF-7 cells, we added ET-RA and ET-RB antagonists (singularly and in combination) to the invasion chambers. Unlike the chemotaxis assays, which showed partial inhibition of MCF-7 migration by both ET-RA and ET-RB antagonists and an additive effect when used in combination, both BQ-123 and BQ-788 could entirely abolish the effect of ET-2 on invasion of MCF-7 cells (Fig. 4D); the effects of BQ-123 and BQ-788 were not additive. Neither BQ-123 nor BQ-788 inhibited macrophage-induced invasion of MCF-7 cells when ETs were not added (data not shown).

ETs Increase MMP Production by Macrophages in Coculture. The increase in tumor cell invasion induced by ET-1 and ET-2 in coculture suggested that the macrophages were producing a soluble factor that aided tumor cell invasion. We analyzed MMP-2 and -9 production by tumor cells and macrophages using zymography. MCF-7 cells produced little or no MMP-2 or -9 with or without stimulation by ETs (Fig. 5A). Cultured in isolation, macrophages produce MMP-2 and -9 , but production was not altered by the addition of ETs (Fig. 5B). However, when cocultured tumor cells and macrophages were treated with ETs, all of the ETs caused an increase in MMP-9 and MMP-2 production (Fig. 5C). The addition of $900 \mu \mathrm{M}$ FN439, a nonspecific MMP inhibitor, to the coculture invasion assay abolished the increase in tumor cell invasion caused by ET-2 (Fig. 5D).

We analyzed MMP (MMP-2, -3, -7, and -9) and TIMP (TIMP-1, TIMP-2, and TIMP-3) mRNA expression by macrophages in coculture by real-time PCR. The addition of ETs or receptor antagonists did
A

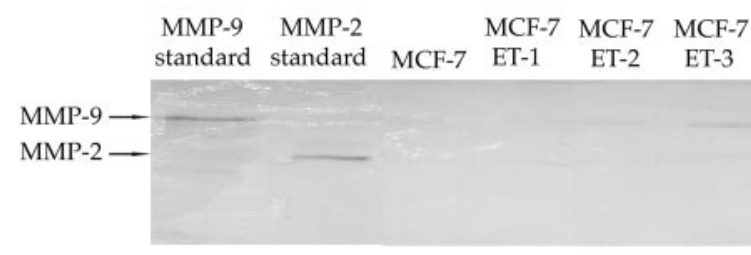

B
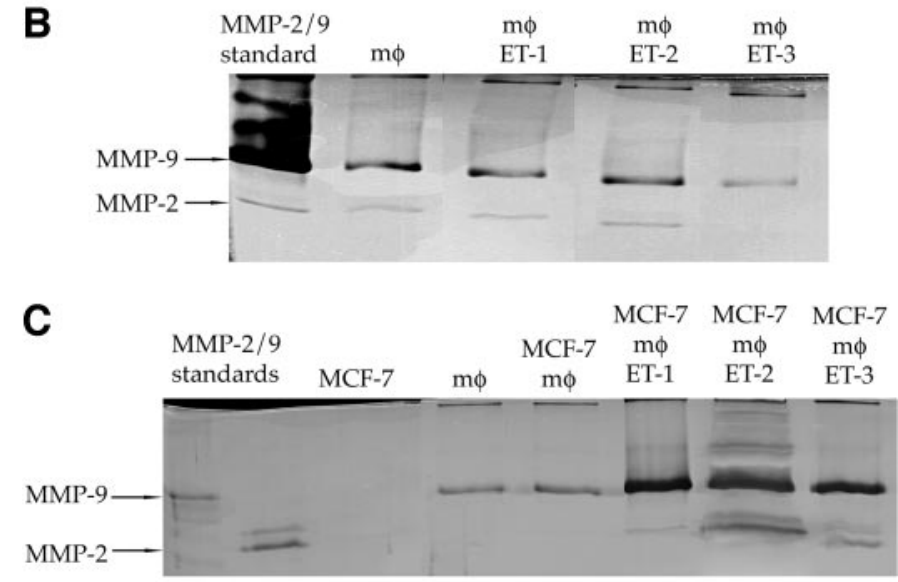

D
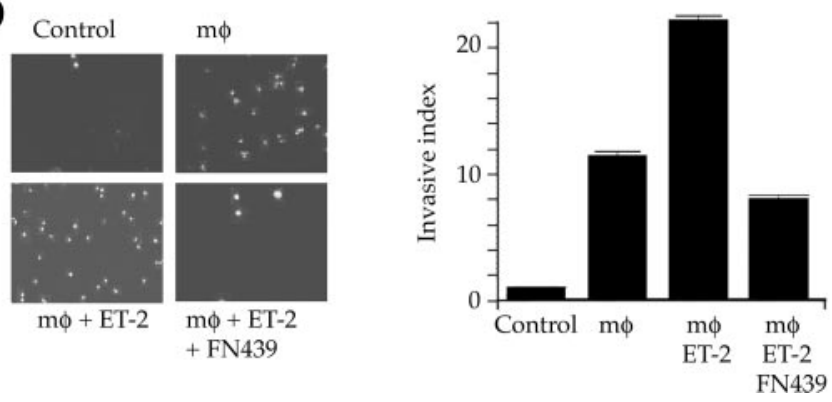

Fig. 5. A, zymograms of medium from endothelin (ET)-stimulated MCF-7 cells. Tumor cells produced undetectable levels of matrix metalloproteinase 2 (MMP-2) and MMP-9, and this was not affected by ET stimulation. $B$, zymograms of medium from ETstimulated macrophages ( $\mathrm{m} \phi$ ET-1, $\mathrm{m} \phi$ ET-2, m $\phi$ ET-3). ETs did not stimulate release of active MMPs into the medium by macrophages. $C$, zymograms of medium from cocultured MCF-7 cells/macrophage. All of the ETs stimulated release of active MMP-9 and MMP-2 into the coculture medium. For all of the zymograms, cells were stimulated with $100 \mathrm{ng} / \mathrm{ml} \mathrm{ET}$ for $96 \mathrm{~h}$ in gelatinase-free medium before the medium was collected. Shown is one representative experiment of three. D, inhibition of ET-induced invasion in $\mathrm{MCF}-7 /$ macrophage coculture by a nonspecific MMP inhibitor. FN439 $(900 \mu \mathrm{M})$ significantly reduced ET-2-stimulated invasion of MCF-7 cells in coculture $(P<0.05)$.

not alter the macrophage mRNA expression of MMPs or TIMPs (data not shown).

ET and ET Receptor Expression Increases from DCIS to IDC to Lymph Node Metastases. In a preliminary study, we compared protein expression of ET and ET receptors in 9 cases of DCIS, 10 IDCs, and 10 lymph node metastases of primary breast carcinomas. ETs were expressed in the cytoplasm, with an increased level in tumor tissue compared with nonneoplastic breast tissue (Fig. 6; Table 1). The percentage of cases with strong positive staining of both ET and ET-RB increased from DCIS cases to IDC to the metastases (Fig. 6; Table 1). We also observed that carcinoma cells that were infiltrating the mammary fat tissue and those in the lymphovascular space tended to stain more intensely for ETs and their receptors than other parts of the same tumor (Fig. 6). It is also of note that in all neoplastic tissue (DCIS, IDC, or lymph node metastases) that showed ET immunopositivity, ET receptor staining, particularly ET-RB, was also observed (Table 2). All of the lymph node metastases showed immunopositivity for both ET and ET-RB.

ET-2 Stimulates ET Receptor Production by Tumor Cells. In view of the fact that ET and ET receptor staining often colocalized in 
Fig. 6. Protein expression of endothelins (ETs) and ET receptors ET-RA and ET-RB in ductal carcinoma in situ (DCIS), invasive ductal carcinoma (IDC), and lymph node metastases (see Tables 1 and 2). Serial sections of DCIS, IDC, and lymph node metastases were immunohistochemically stained for ETs (using an antibody that recognizes all ET isoforms), ET-RA, and ET-RB. Negative control sections were probed with antibodies for unexpressed proteins (e.g., gastrin, shown), or serum was used in place of the primary antibody. Sections were stained for ETs in the left panels, ET-RA in the center panels, and ET-RB in the right panels. Row 1, DCIS; row 2, IDC; row 3, cells infiltrating fatty tissue; row 4, lymphovascular invasion; row 5, a metastatic lymph node.

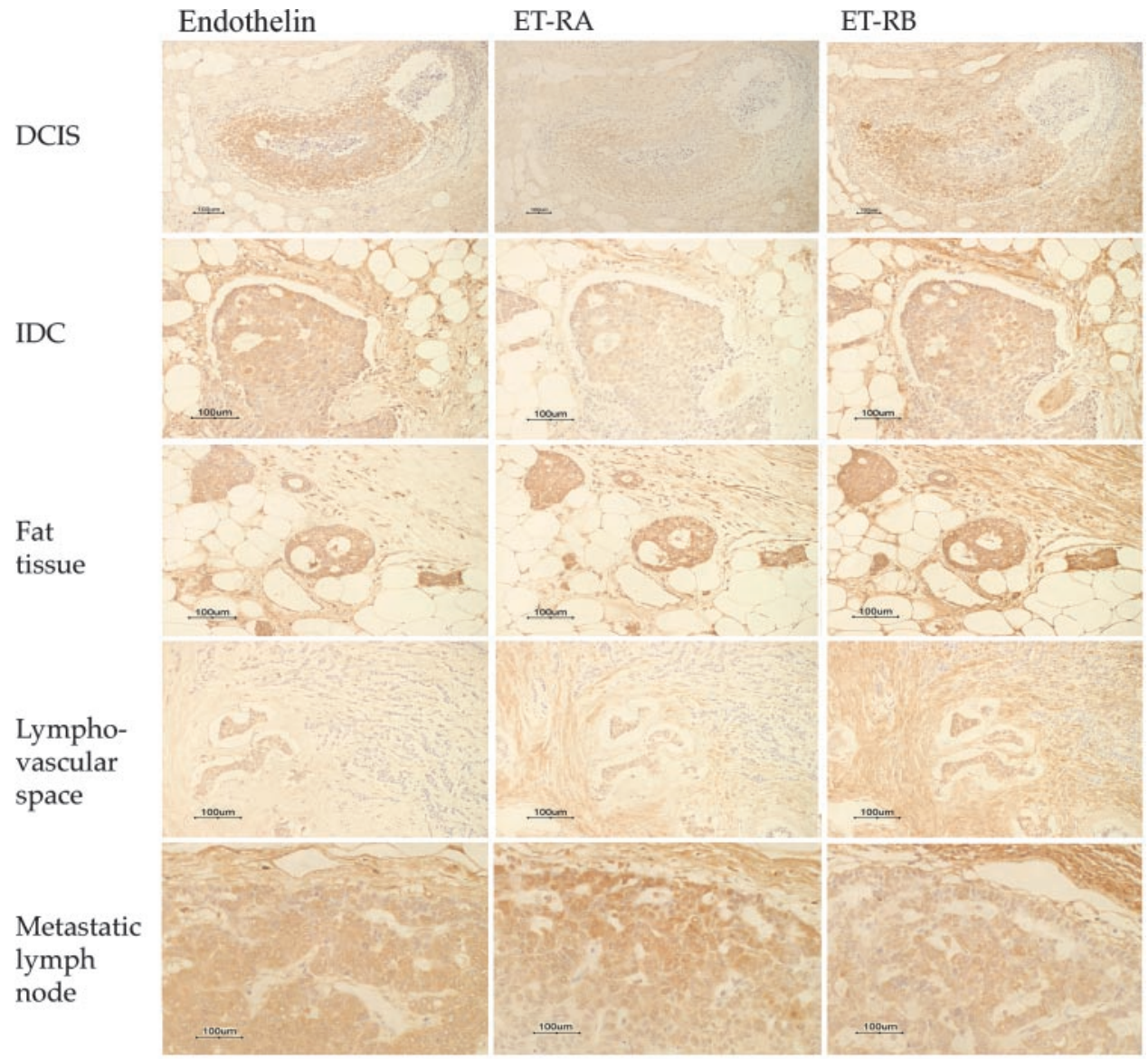

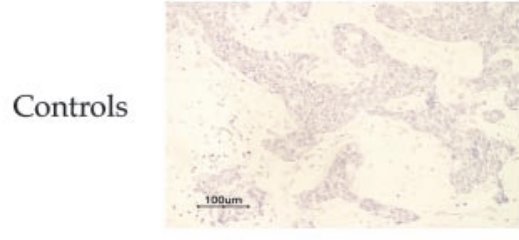

Antibody control

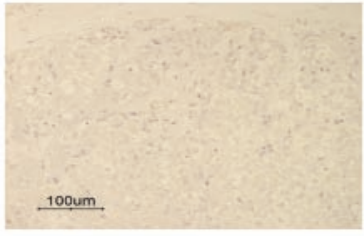

Rabbit serum control

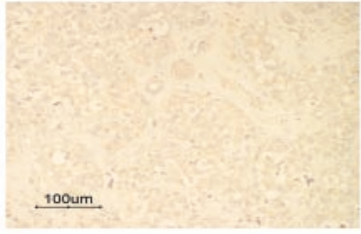

Sheep serum control vivo and in view of the potential autocrine role(s) of ET-2 in breast neoplasia, we analyzed whether ET-2 induced ET receptor production by MCF-7 cells in vitro. Cells stimulated with $100 \mathrm{ng} / \mathrm{ml}$ ET-2 showed a rapid and sustained (1-24 h) increase in the production of ET-RB (Fig. 7); ET-RA production by MCF-7 cells exposed to ET-2 was increased by $24 \mathrm{~h}$.

Table 1 Endothelin and endothelin receptor (ET-RA, ET-RB) protein expression in ductal carcinoma in situ (DCIS), invasive ductal carcinoma (IDC), and metastatic lymph nodes ( $L N$ Met)

The number and percentage of cases positively immunostained for endothelin and ET-RB increased from DCIS to IDC and IDC to LN Met. Numbers in bold indicate total number of positive $(+\mathrm{ve})$ samples, and numbers in parentheses are the percentages of positive cases. Positivity was assessed by the percentage of tumor cell immunostaining and divided into weak (1-25\%) and strong ( $>25 \%$ tumor cell positivity).

\begin{tabular}{|c|c|c|c|c|c|c|c|}
\hline & \multirow[b]{2}{*}{$\begin{array}{c}\text { Total } \\
\text { samples }\end{array}$} & \multicolumn{2}{|c|}{ Endothelin staining } & \multicolumn{2}{|c|}{ ET-RA staining } & \multicolumn{2}{|c|}{ ET-RB staining } \\
\hline & & $\begin{array}{c}\text { Total } \\
+ \text { ve }(\%)\end{array}$ & $\begin{array}{c}\text { Strong } \\
+ \text { ve }(\%)\end{array}$ & $\begin{array}{c}\text { Total } \\
+ \text { ve }(\%)\end{array}$ & $\begin{array}{c}\text { Strong } \\
+ \text { ve }(\%)\end{array}$ & $\begin{array}{c}\text { Total } \\
+ \text { ve }(\%)\end{array}$ & $\begin{array}{l}\text { Strong } \\
+ \text { ve }(\%)\end{array}$ \\
\hline DCIS & 9 & $5(56)$ & $3(33)$ & $6(67)$ & $0(0)$ & $5(56)$ & $3(33)$ \\
\hline IDC & 10 & $9(90)$ & $5(50)$ & $3(30)$ & $0(0)$ & $7(70)$ & $4(40)$ \\
\hline LN Met & 10 & $10(100)$ & $6(60)$ & $3(30)$ & $0(0)$ & $10(100)$ & $9(90)$ \\
\hline
\end{tabular}

\section{DISCUSSION}

Many tumor-linked factors have been implicated in the development and progression of invasion and there has recently been interest in the role of ET-1/ET-RA, particularly in ovarian and prostate cancer metastasis $(14,23)$. Normal breast tissue expresses little ET or ET$\mathrm{RB}$, but intratumoral hypoxia $(24,25)$ induces expression of ET-2 and both ET receptors by tumor cells (6). We have now shown that ETs induce a chemotactic and invasive phenotype in breast tumor cells in vitro, particularly when tumor cells are cocultured with macrophages. Furthermore, data from the preliminary immunohistochemical study indicates that ET and ET receptor expression increases from normal breast to DCIS to IDC to metastatic deposits. This is the first time a possible role for ETs in breast cancer invasion has been identified and also the first time that ET-2/ET-RB has been implicated in invasion/ progression.

It is interesting that tumor cells migrate strongly toward ET-2; however, it is difficult to understand whether this may have a broader role in metastasis. Physiologically, ET-2 expression is limited to the kidney and the intestine and is not released systemically in any great concentration (1). Thus, it is unlikely that chemotaxis toward ET-2 by 
Table 2 Endothelin and receptor (ET-RA, ET-RB) protein coexpression in ductal carcinoma in situ (DCIS), invasive ductal carcinoma (IDC), and lymph node metastases ( $L N$ Met)

In tumors that showed endothelin immunopositivity, expression of ET-RA and/or ET-RB was also often found. The correlation between endothelin immunopositivity and ET-RB positivity was strongest. LN Met showed 100\% immunopositivity for endothelin and ET-RB. These figures include all immunopositives (weak and strong positives).

\begin{tabular}{|c|c|c|c|c|c|c|c|}
\hline & \multirow{2}{*}{$\begin{array}{c}\text { Total } \\
\text { samples }\end{array}$} & \multicolumn{2}{|c|}{ Endothelin } & \multicolumn{2}{|c|}{$\begin{array}{l}\text { Endothelin and (ET- } \\
\text { RA and/or -RB) }\end{array}$} & \multicolumn{2}{|c|}{$\begin{array}{c}\text { Endothelin and } \\
\text { ET-RB }\end{array}$} \\
\hline & & Positive & $(\%)$ & Positive & $(\%)$ & Positive & $(\%)$ \\
\hline DCIS & 9 & 5 & (56) & 5 & (56) & 4 & (44) \\
\hline IDC & 10 & 9 & (90) & 6 & (60) & 6 & (60) \\
\hline LN Met & 10 & 10 & (100) & 10 & (100) & 10 & (100) \\
\hline
\end{tabular}

breast tumor cells could explain organ-selective metastases, although breast cancers may metastasize to the kidney (26). It is also worth noting that ET-1 is expressed at several additional sites of breast cancer metastasis including bone (27).

It is conceivable, however, that migration toward ET-2 or ET-1 has an impact within the tumor, such that cells mass within hypoxic areas and become more aggressive because of exposure to ETs or by selective pressure of hypoxia. It is also possible that tumor cells may use ETs to migrate toward blood vessels, in which ET-1 is likely to be expressed (28), and thus can begin the process of intravasation. The high levels of ET and ET receptor expression by tumor cells within blood vessels and the high levels of gradient-independent invasion induced by ET-1 or ET-2 suggests that ET/ET receptor interactions have a role in intravasation and the preliminary steps of invasion and metastasis.

Systemically, it may be of more significance that treating tumor cells with ET-2 leads to increased chemotaxis toward the chemokines CXCL12 and CCL21, which are expressed by the lymph nodes and other sites of breast cancer metastasis. We were not able to detect an up-regulation of chemokine receptors on MCF-7 cells when stimulated by ET-2, and, thus, it may be that ET-2 impinges on the signaling pathways to amplify the response of tumor cells to chemokines.

Similar to macrophages (11), the preliminary steps in ET signaling in tumor cells is typical of chemokine signaling (29). Despite the action of ET-2 during cellular stress (6), ET-2 does not signal via the stress-activated p38 or SAPK/JNK pathways, but via the MEK1/2 and p42/p44 MAPK pathway. ET-2 signaling also involves activation of the G-protein-coupled ET receptors and an influx of extracellular calcium. Although ET-2 signaling in tumor cells has parallels with ET-2/ET-RB signaling in macrophages (11), it is clear that there are several differences. Macrophage chemotaxis toward ET-2 involves solely ET-RB, whereas chemotaxis and invasion of breast tumor cells involved both ET-RA and ET-RB. Chemotaxis toward ET-2 of both cell types can be inhibited by PD98059 or PTX, yet only tumor cells can migrate toward ET-2 under hypoxia. Both ET-2 and hypoxia increase ET receptor expression by tumor cells (6), and this may potentiate hypoxic migration. There may also be a role for ET-RA in hypoxic migration, which is expressed by tumor cells but not by macrophages (11). The chemotactic differences could also be due to the time of the chemotaxis assays; macrophage migration under hypoxia is inhibited rapidly by a short-term induction of MKP-1 (30), whereas tumor cells are allowed to migrate over a period of several hours.

Although the complete abolition of ET-induced chemotaxis required the inhibition of both receptors, ET-induced invasion of MCF-7 cells in coculture could be abolished by either receptor antagonist. This may be due to the complex processes and interactions that occur during invasion in macrophage/tumor cell coculture; individual elements of the invasion process may be mediated by one or other ET receptor allowing abolition of invasion by one receptor antagonist alone. The relative contributions of ET-RA and ET-RB signaling when tumor cells are stimulated by ET-2 are yet to be elucidated and may help us to understand the differences between macrophage and tumor cell signaling pathways.

Macrophages, which colocalize with ETs in breast cancer (11), increased MMP production in response to ETs, thus facilitating tumor cell invasion. Hagemann et al ${ }^{6}$ showed that the increase in MMP production in macrophage/tumor cell cocultures was due to the macrophages and not to the tumor cells. The inhibition of the MMPs was sufficient to prevent MCF-7 cell invasion, which shows the critical role of these enzymes in invasion. However, all of the ETs could increase production of MMP-2 and -9 by macrophages, yet ET-3 did not increase the invasion of tumor cells. This indicates that additional factors produced by cocultured cells and stimulated (or inhibited) by ETs, distinct from MMP-9 and MMP-2, must also play a role in tumor cell invasion. It is probable that there is a complex interaction between ET expression, hypoxia, and tumor-associated macrophages within the breast tumor such that invasion is increased. However, additional interactions may occur such as paracrine ET stimulation of tumor endothelial cells.

As well as the paracrine actions of ETs in breast tumors, those neoplasia that expressed ET in vivo also expressed the ET receptors, which may indicate an autocrine loop. We recently proposed an autocrine survival function for ET-2/ET-RB binding in breast carcinoma cells under hypoxic conditions (6). All of the human cell lines expressed ET-1 and ET-2 in vitro as well as one or more ET receptor; furthermore, production of the ET receptors by tumor cells in vitro was stimulated by ET-2. These data support an autocrine action of ETs to increase the invasiveness of tumor cells.

Examination of the expression of ETs and their receptors in a large number of breast carcinomas and metastatic deposits, and correlation with macrophage infiltrate, is now required to determine whether there is a correlation between ET/ET receptor expression and grade (or currently used conventional prognostic factors) and prognosis. Such studies may also indicate whether treatment of breast cancer and its metastases with ET receptor antagonists may have a therapeutic benefit. Because ETs stimulates not only breast cancer cell invasion but also chemotaxis toward chemokines, it may be that combined treatment with both ET receptor antagonists and chemokine receptor antagonists may potentially prevent breast cancer metastases better

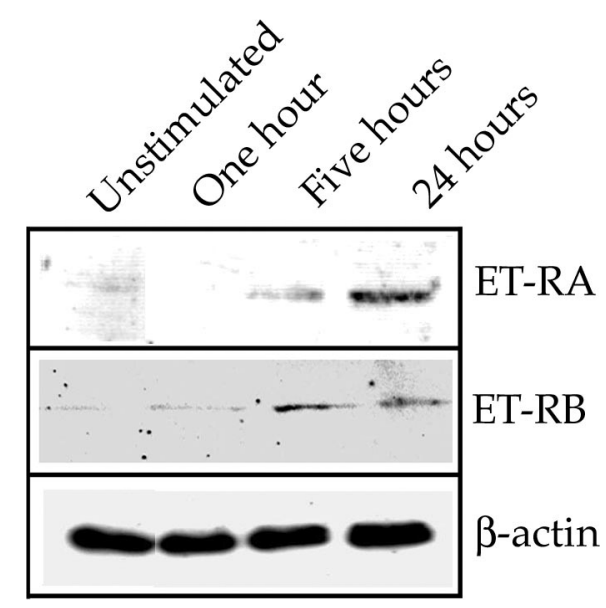

Fig. 7. Western blots of protein extracts from MCF-7 cells stimulated with endothelin (ET)-2. MCF-7 cells were serum-starved for $24 \mathrm{~h}$ before being stimulated with ET-2 for 0-24 h, and blots were probed for ET receptors ET-RA and ET-RB. Stimulation with ET-2 led to increased ET-RA protein expression by $24 \mathrm{~h}$, whereas ET-RB protein was increased by one $\mathrm{h}$ and remained increased at $24 \mathrm{~h}$. Blots were probed for $\beta$-actin to control for protein loading. Shown is one experiment of three. 
than do single treatments alone. Atrasentan, a selective ET-RA antagonist, is already in clinical trials for prostate cancer (31) and may be of therapeutic benefit for the treatment of IDC of the breast.

Up-regulation of ET-2 and its receptors by hypoxia (6), together with this data implying a role for ET-2 in invasion, may be one factor that explains why hypoxic tumors are more aggressive than nonhypoxic ones (10) and why hypoxia increases carcinoma cell invasion (32).

Not only is this the first time that a role for ET-2 or ET-RB in tumor cell invasion and metastasis has been proposed but it is also the first time the role of ET-stimulated macrophages in tumor cell invasion has been studied. The data showing ET-associated induction of MMP release by macrophages in coculture and increased chemotactic/invasive capacity of tumor cells together with increasing expression of ETs and ET receptor protein from DCIS to IDC to tumors in metastatic lymph nodes support a role for ET expression in progression and metastasis.

\section{REFERENCES}

1. Levin ER. Endothelins. N Engl J Med 1995;333:356-63.

2. Masaki T. The endothelin family: an overview. J Cardiovasc Pharmacol 2000;35: S3-5.

3. Pollock DM, Keith TL, Highsmith RF. Endothelin receptors and calcium signaling. FASEB J 1995;9:1196-204.

4. Haendler B, Hechler U, Schleuning WD. Molecular cloning of human endothelin (ET) receptors ETA and ETB. J Cardiovasc Pharmacol 1992;20:S1-4.

5. Nelson J, Bagnato A, Battistini B, Nisen P. The endothelin axis: emerging role in cancer. Nat Rev Cancer 2003;3:110-6.

6. Grimshaw MJ, Naylor S, Balkwill FR. Endothelin-2 Is a Hypoxia-induced autocrine survival factor for breast tumor cells. Mol Cancer Ther 2002;1:1273-81.

7. Ishikawa K, Ihara M, Noguchi K, et al. Biochemical and pharmacological profile of a potent and selective endothelin B-receptor antagonist, BQ-788. Proc Natl Acad Sci USA 1994;91:4892-6.

8. Ihara M, Noguchi K, Saeki T, et al. Biological profiles of highly potent novel endothelin antagonists selective for the ETA receptor. Life Sci 1992;50:247-55.

9. Ueno T, Toi M, Saji H, et al. Significance of macrophage chemoattractant protein-1 in macrophage recruitment, angiogenesis, and survival in human breast cancer. Clin Cancer Res 2000;6:3282-9.

10. Dachs GU, Tozer GM. Hypoxia modulated gene expression: angiogenesis, metastasis and therapeutic exploitation. Eur J Cancer 2000;36:1649-60.

11. Grimshaw MJ, Wilson JL, Balkwill FR. Endothelin-2 is a macrophage chemoattractant: implications for macrophage distribution in tumors. Eur J Immunol 2002;32: 2393-400.
12. Peacock AJ, Dawes KE, Shock A, Gray AJ, Reeves JT, Laurent GJ. Endothelin-1 and endothelin-3 induce chemotaxis and replication of pulmonary artery fibroblasts. Am J Respir Cell Mol Biol 1992;7:492-9.

13. Elferink JG, de Koster BM. Modulation of human neutrophil chemotaxis by the endothelin-B receptor agonist sarafotoxin S6c. Chem Biol Interact 1996;101:165-74.

14. Rosano L, Varmi M, Salani D, et al. Endothelin-1 induces tumor proteinase activation and invasiveness of ovarian carcinoma cells. Cancer Res 2001;61:8340-6.

15. Berry P, Burchill S. Endothelins may modulate invasion and proliferation of Ewing's sarcoma and neuroblastoma. Clin Sci (Lond) 2002;103 Suppl 48:S322-6.

16. Ihara M, Ishikawa K, Fukuroda T, et al. In vitro biological profile of a highly potent novel endothelin (ET) antagonist BQ-123 selective for the ETA receptor. J Cardiovasc Pharmacol 1992;20 Suppl 12:S11-4.

17. Dias S, Boyd R, Balkwill F. IL-12 regulates VEGF and MMPs in a murine breast cancer model. Int J Cancer 1998;78:361-5.

18. Traish AM, Moran E, Daley JT, de las Morenas A, Saenz de Tejada I. Monoclonal antibodies to human endothelin-1: characterization and utilization in radioimmunoassay and immunocytochemistry. Hybridoma 1992;11:147-63.

19. Furuya S, Hiroe T, Ogiso N, Ozaki T, Hori S. Localization of endothelin-A and -B receptors during the postnatal development of rat cerebellum. Cell Tissue Res 2001;305:307-24.

20. Alessi DR, Cuenda A, Cohen P, Dudley DT, Saltiel AR. PD 098059 is a specific inhibitor of the activation of mitogen-activated protein kinase kinase in vitro and in vivo. J Biol Chem 1995;270:27489-94.

21. Heinroth-Hoffmann I, Vogelsang M, Schiewe P, et al. Mechanism of ET(A)-receptor stimulation-induced increases in intracellular Ca2+ in SK-N-MC cells. Br J Pharmacol 1998;125:1202-11.

22. Muller A, Homey B, Soto $\mathrm{H}$, et al. Involvement of chemokine receptors in breast cancer metastasis. Nature (Lond.) 2001;410:50-6.

23. Guise TA, Yin JJ, Mohammad KS. Role of endothelin-1 in osteoblastic bone metastases. Cancer (Phila.) 2003;97:779-84.

24. Vaupel P, Schlenger K, Knoop C, Hockel M. Oxygenation of human tumors: evaluation of tissue oxygen distribution in breast cancers by computerized $\mathrm{O}_{2}$ tension measurements. Cancer Res 1991;51:3316-22.

25. Runkel S, Wischnik A, Teubner J, Kaven E, Gaa J, Melchert F. Oxygenation of mammary tumors as evaluated by ultrasound-guided computerized- $\mathrm{pO}_{2}$-histography. Adv Exp Med Biol 1994;345:451-8.

26. Yeatman TJ, Nicolson GL. Molecular basis of tumor progression: mechanisms of organ-specific tumor metastasis. Semin Surg Oncol 1993;9:256-63.

27. Chandel NS, Maltepe E, Goldwasser E, Mathieu CE, Simon MC, Schumacker PT. Mitochondrial reactive oxygen species trigger hypoxia-induced transcription. Proc Natl Acad Sci USA 1998;95:11715-20.

28. Bagnato A, Spinella F. Emerging role of endothelin-1 in tumor angiogenesis. Trends Endocrinol Metab 2002;14:44-50.

29. Olson TS, Ley K. Chemokines and chemokine receptors in leukocyte trafficking. Am J Physiol Regul Integr Comp Physiol 2002;283:R7-28.

30. Grimshaw MJ, Balkwill FR. Inhibition of monocyte and macrophage chemotaxis by hypoxia and inflammation-a potential mechanism. Eur J Immunol 2001;31:480-9.

31. Norman P. Atrasentan Abbott. Curr Opin Investig Drugs 2002;3:1240-8.

32. Graham CH, Forsdike J, Fitzgerald CJ, Macdonald-Goodfellow S. Hypoxia-mediated stimulation of carcinoma cell invasiveness via upregulation of urokinase receptor expression. Int J Cancer 1999;80:617-23. 


\section{A Role for Endothelin-2 and Its Receptors in Breast Tumor Cell Invasion}

Matthew J. Grimshaw, Thorsten Hagemann, Ayse Ayhan, et al.

Cancer Res 2004;64:2461-2468.

Updated version Access the most recent version of this article at:

http://cancerres.aacrjournals.org/content/64/7/2461

Cited articles This article cites 32 articles, 7 of which you can access for free at:

http://cancerres.aacrjournals.org/content/64/7/2461.full\#ref-list-1

Citing articles This article has been cited by 10 HighWire-hosted articles. Access the articles at:

http://cancerres.aacrjournals.org/content/64/7/2461.full\#related-urls

E-mail alerts Sign up to receive free email-alerts related to this article or journal.

Reprints and To order reprints of this article or to subscribe to the journal, contact the AACR Publications Subscriptions Department at pubs@aacr.org.

Permissions To request permission to re-use all or part of this article, use this link http://cancerres.aacrjournals.org/content/64/7/2461.

Click on "Request Permissions" which will take you to the Copyright Clearance Center's (CCC)

Rightslink site. 